\title{
Direct Growth of Copper Oxide Films on Ti Substrate for Nonenzymatic Glucose Sensors
}

\author{
Xiaoxu Ji, Aihua Wang, and Qinghuai Zhao \\ School of Physics and Electronic Engineering, Nanyang Normal University, Nanyang 473061, China \\ Correspondence should be addressed to Xiaoxu Ji; xxji@nynu.edu.cn
}

Received 8 June 2014; Accepted 9 June 2014; Published 25 June 2014

Academic Editor: Xiang Wu

Copyright (C) 2014 Xiaoxu Ji et al. This is an open access article distributed under the Creative Commons Attribution License, which permits unrestricted use, distribution, and reproduction in any medium, provided the original work is properly cited.

Copper oxide $(\mathrm{CuO})$ films directly grown on Ti substrate have been successfully prepared via a hydrothermal method and used to construct an amperometric nonenzymatic glucose sensor. XRD and SEM were used to characterize the samples. The electrochemical performances of the electrode for detection of glucose were investigated by cyclic voltammetry and chronoamperometry. The CuO films based glucose sensors exhibit enhanced electrocatalytic properties which show very high sensitivity $\left(726.9 \mu \mathrm{A} \mathrm{mM} \mathrm{m}^{-1} \mathrm{~cm}^{-2}\right)$, low detection limit $(2 \mu \mathrm{M})$, and fast response $(2 \mathrm{~s})$. In addition, reproducibility and long-term stability have been observed. Low cost, convenience, and biocompatibility make the $\mathrm{CuO}$ films directly grown on Ti substrate electrodes a promising platform for amperometric nonenzymatic glucose sensor.

\section{Introduction}

Reliable and fast determination of glucose is important in areas such as clinical diagnostics [1], biotechnology [2], and environmental and food chemistry [3], so the development of electrochemical glucose sensors has received continuous interest. Glucose oxidase $\left(\mathrm{GO}_{x}\right)$ has been widely used to construct various amperometric biosensors for glucose detection due to its high sensitivity and selectivity to glucose [4]. However, $\mathrm{GO}_{x}$-based biosensors suffer from problems because of the disadvantages of the enzyme-modified electrodes, such as instability, high cost, complicated immobilization process, the requirement of low temperature storage, and their $\mathrm{PH}$ and toxic environment [5-8]. To resolve this problem, many attempts have been made to develop nonenzymatic glucose sensors in recent years. For example, the direct electrocatalytic oxidation of glucose on electrodes modified with metal nanoparticles has been explored in the development of nonenzymatic glucose sensors because it shows good performances through increasing the surface area and enhancing the mass transport and catalysis [914]. However, metallic nanoparticles based electrodes usually suffer from the stable problems [15] or high cost [16], which hinders their practical applications. Hence, it is important to develop a fast, sensitive, highly selective, stable, and inexpensive nonenzymatic glucose sensor. In contrast, metal oxides based glucose sensors have been widely investigated for nonenzymatic detecting due to their good stability and low cost.

$\mathrm{CuO}$, as a $p$-type semiconductor with a narrow band gap of $1.2 \mathrm{eV}$, has been widely studied because of its numerous applications in catalysis, gas sensors, lithium-ion battery, and field transistors [17-20]. Meanwhile, nanostructured $\mathrm{CuO}$ is a very economic functional material which is promising in the development of nonenzymatic glucose sensors because of its highly specific surface area, good electrochemical activity, and the possibility of promoting electron transfer reactions at a lower overpotential [21]. It is well known that the microstructures (crystal size, aspect ratio, density, etc.) and morphologies of metal oxide nanoparticles play a determining role for the activity, selectivity, and stability in a catalytic process. Thus, a variety of $\mathrm{CuO}$ nanomaterials with different morphologies including nanowires [22], nanospheres [23], nanosheets [24], and flower-like nanostructures [25] have been synthesized in order to achieve enhanced performance for the monitoring and detection of glucose. In comparison to nanostructures, structured $\mathrm{CuO}$ films could avoid the drawbacks of complex fabrication processed, harsh modification 
conditions for electrodes, expensive glassy carbon electrode, and possible decline of electron transfer rate between isolated nanostructures and electrodes. Recently, $\mathrm{CuO}$ nanostructures (nanosheets [26], nanobelts [27], and nanowalls [28]) on $\mathrm{Cu}$ foils directly applied for glucose sensing have been explored. To the best of our knowledge, $\mathrm{CuO}$ grown directly on electric and biocompatible substrate, which acts as electrode for detection of glucose have been rarely reported. Here, we design a new $\mathrm{CuO}$ film on $\mathrm{Ti}$ substrate $(\mathrm{CuO} / \mathrm{Ti})$ electrode by effectively taking advantage of the conductivity and biocompatibility of the substrate. The $\mathrm{CuO} / \mathrm{Ti}$ electrode, fabricated simply by a template-free hydrothermal method, can be an inexpensive and high-performance alternative to conventional $\mathrm{CuO}$ electrodes for use in biosensors. There is no need to use extra binders or other classical electrodes such as glassy carbon and Au electrode. We investigated the application of this new electrode for glucose determination. In addition, we demonstrated that the fabricated glucose biosensor exhibited high sensitivity, fast response, an appropriate linear range, and good stability. The good analytical performance, low cost, and one-step preparation method make this electrode material promising for the development of a nonenzymatic glucose sensor.

\section{Experimental Details}

2.1. Reagents and Materials. $\mathrm{Cu}\left(\mathrm{NO}_{3}\right)_{2} \cdot 3 \mathrm{H}_{2} \mathrm{O}, \mathrm{NaOH}$, urea, and glucose are purchased from Shanghai Chemical Co. (China). All chemicals used are analytical grade and are used as received without further purification. The titanium foil is of $99 \%$ purity. Glucose solution containing various concentrations is freshly prepared daily. All aqueous solutions are prepared in distilled water.

\subsection{Preparation of $\mathrm{CuO}$ Films on Ti Substrate Electrodes.} In a typical process, $1 \mathrm{~m} \mathrm{~mol} \mathrm{Cu}\left(\mathrm{NO}_{3}\right)_{2} \cdot 3 \mathrm{H}_{2} \mathrm{O}$ and $10 \mathrm{~m} \mathrm{~mol}$ urea were dissolved in $80 \mathrm{~mL}$ distilled water to form a blue transparent solution. Then, the solution was transferred into a Teflon-lined autoclave (a piece of $\mathrm{Ti}$ substrate washed by hydrofluoric acid/water was placed upright in the autoclave). After that, the autoclave was placed in an electric oven at $120^{\circ} \mathrm{C}$ for $12 \mathrm{~h}$. Lastly, the substrate was washed with distilled water and dried at room temperature. In this way, $\mathrm{CuO}$ films could be fabricated on the Ti substrate by a simple method with low cost. This opened up the possibility of large-scale preparation of the novel probe which can be applied as the electrode for a glucose sensor.

\subsection{Sample Characterizations and Electrochemical Test. The} film was characterized using powder X-ray diffraction (XRD, $\mathrm{Y}-2000)$ with $\mathrm{Cu} \mathrm{K} \alpha$ radiation $(\lambda=1.5418 \AA)$ and scanning electron microscopy (SEM, JSM-6700F). All electrochemical measurements were performed on a CHI 660C electrochemical workstation (Shanghai, China) with a conventional three-electrode system composed of a platinum auxiliary, a saturated calomel electrode (SCE) reference, and $\mathrm{CuO}$ films grown on $\mathrm{Ti}$ substrate as working electrodes. All electrochemical experiments were performed in $0.1 \mathrm{M} \mathrm{NaOH}$ solutions under continuous stirring at room temperature. Glucose concentration was controlled by addition as desired.

\section{Results and Discussion}

3.1. Morphology and Structure of Obtained Sample. XRD pattern of the as-obtained sample is displayed in Figure 1(a). Except for the peaks originating from the Ti substrate, others are the peaks of monoclinic phase of $\mathrm{CuO}$ (JCPDS number 89-5899) with lattice constants of $a=0.469 \mathrm{~nm}, b=$ $0.342 \mathrm{~nm}$, and $c=0.513 \mathrm{~nm}$. The major sharp peaks at $35.5^{\circ}$ and $38.7^{\circ}$ are attributable to the (002) and (200) plane, respectively, indicating pure phase monoclinic crystallites. Figure 1(b) illustrates a typical optical image of $\mathrm{CuO}$ film grown on Ti substrate. As it is obvious from the image, the prepared $\mathrm{CuO}$ film is uniform on $\mathrm{Ti}$ substrate on a large scale. The typical morphology of the as-obtained $\mathrm{CuO}$ film, as observed by SEM, is illustrated in Figures 1(c) and 1(d) at different magnifications. Figure 1(c) is the low-magnification SEM image. It can be seen that the substrate is absolutely covered by uniform maize cob-like $\mathrm{CuO}$ nanostructures. From Figure 1(d), it is displayed that maize cob-like nanostructure is composed of many small nanoparticles, which has the potential to provide large surface area and high surface energy for catalytic reaction. The mechanism for the direct growth of $\mathrm{CuO}$ film on $\mathrm{Ti}$ substrate could be ascribed to the uniform roughness of etched Ti substrate surface. Roughness of substrate surface is especially valuable to the reduction of binding energy between seeds and substrate [29]. Therefore, it is more convenient for the nucleation and adhesion of seeds to be done on rough surface than on smooth surface.

3.2. Electrochemical Activities. The electrocatalytic activity of the $\mathrm{CuO} / \mathrm{Ti}$ electrode towards the oxidation of glucose in alkaline solution was demonstrated by voltammograms $\left(\mathrm{CV}_{\mathrm{s}}\right)$. The $\mathrm{CV}_{\mathrm{s}}$ of the $\mathrm{CuO} / \mathrm{Ti}$ electrode were conducted in $0.1 \mathrm{M} \mathrm{NaOH}$ solution in the presence and absence of glucose, respectively, at a scan rate of $100 \mathrm{mV} / \mathrm{s}$.

As shown in Figure 2, only small background current and no peak are observed for the pure Ti electrode, while a dramatic increase of current signal with a wave potential of about $+0.58 \mathrm{~V}$ is observed when the $\mathrm{CuO} / \mathrm{Ti}$ electrode is used, which should correspond to a $\mathrm{Cu}(\mathrm{II}) / \mathrm{Cu}$ (III) redox couple. This result is similar to precious reports [30, 31]. This indicates that $\mathrm{CuO}$ nanostructures have greatly improved the performance of the electrode and increased the electrocatalytic ability towards glucose oxidation, which may be attributed to their large surface, high surface energy, and enhanced electron transfer ability [23]. The good electrochemical ability and easy fabrication method make the as-obtained $\mathrm{CuO} / \mathrm{Ti}$ electrode an excellent electrochemical sensing platform for glucose detection. Figure 3(a) displays a typical amperometric response curve of the $\mathrm{CuO} / \mathrm{Ti}$ electrode to the successive addition of glucose at an applied potential of $+0.58 \mathrm{~V}$ in $0.1 \mathrm{M} \mathrm{NaOH}$ recorded at an interval of $50 \mathrm{~s}$. A well-defined, stable, and fast amperometric response could be observed with successive addition of glucose to $\mathrm{NaOH}$. As the 


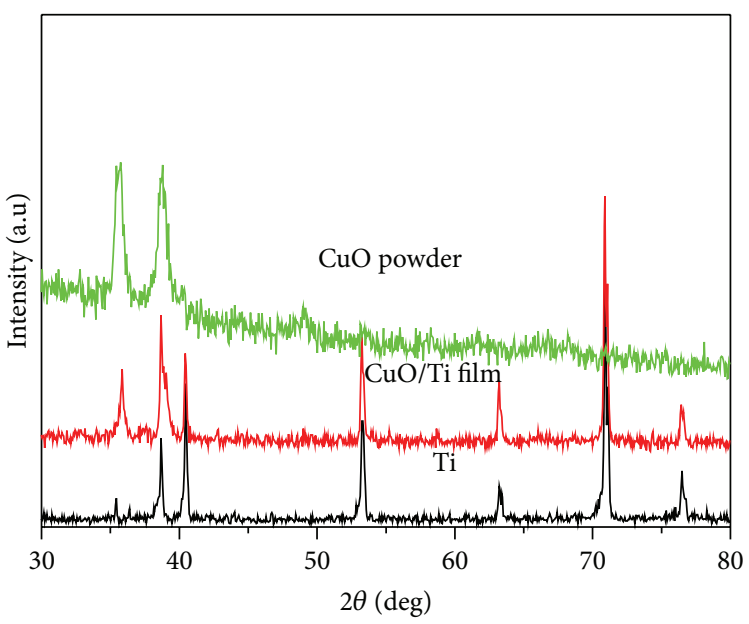

(a)

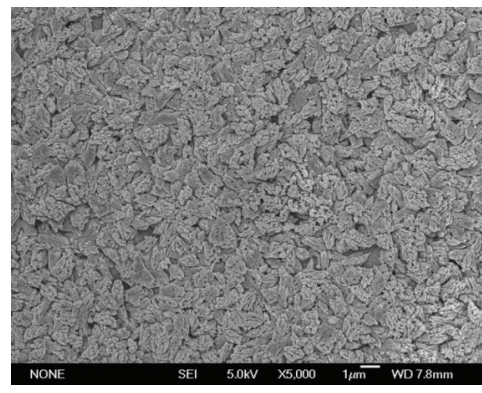

(c)

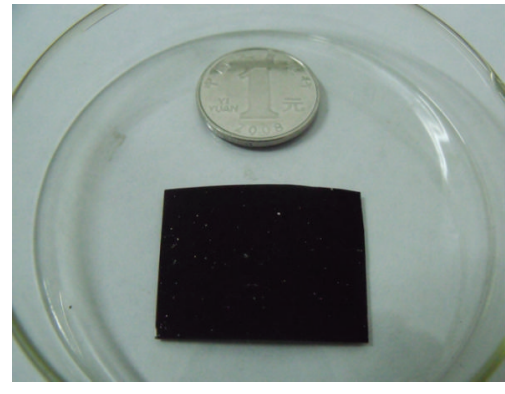

(b)

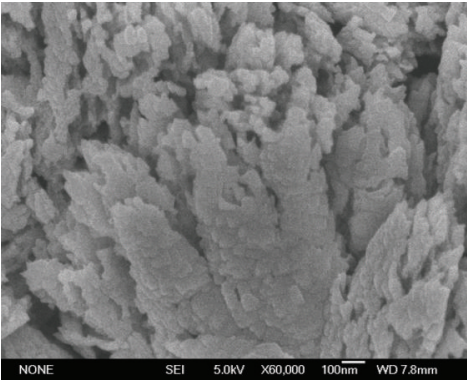

(d)

FIgURE 1: (a) XRD pattern of Ti substrate, $\mathrm{CuO}$ film on Ti substrate, and $\mathrm{CuO}$ powder, (b) optical image, (c) low-magnification, and (d) high-magnification SEM images of as-prepared $\mathrm{CuO}$ film on Ti substrate.

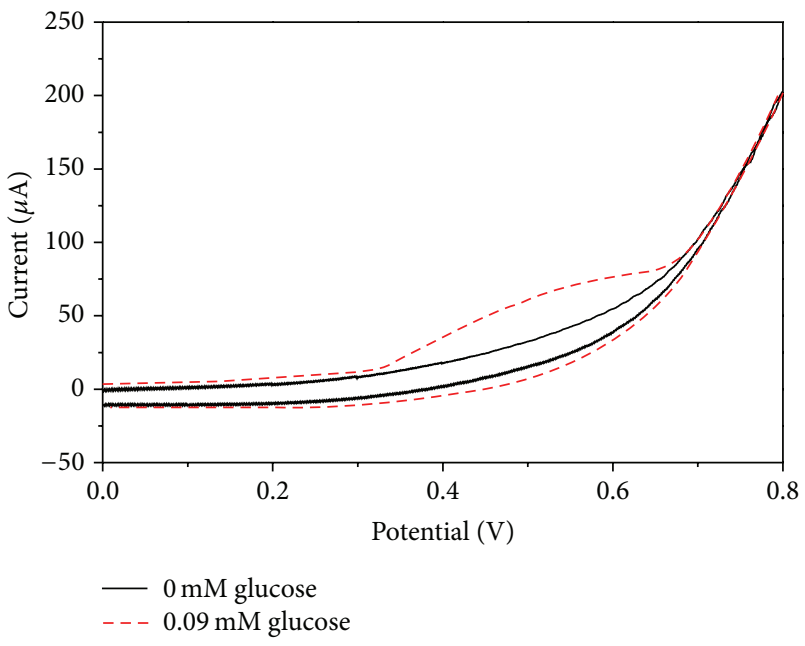

FIgURE 2: Cyclic voltammograms of $\mathrm{CuO}$ film electrode in absence and presence of glucose in $0.1 \mathrm{M} \mathrm{NaOH}$ solution at a scan rate of $100 \mathrm{mV} / \mathrm{s}$.

glucose was injected, the biosensor yields a typical steadystate amperometric response. The average time required to reach the stable response is less than $2 \mathrm{~s}$, demonstrating a fast current response to glucose. Meanwhile, as can be seen from Figure 3(b), the $\mathrm{CuO} / \mathrm{Ti}$ electrode gives a linear dependence range from $5 \mu \mathrm{M}$ to $1.6 \mathrm{mM}$ of glucose, a remarkably high sensitivity of $726.9 \mu \mathrm{A} \mathrm{mM}^{-1} \mathrm{~cm}^{-2}$, and a low detection limit of $2 \mu \mathrm{M}$.

Although the direct electrocatalytic oxidation of glucose at copper-based electrode remains ambiguous till now, the $\mathrm{Cu}^{2+} / \mathrm{Cu}^{3+}$ redox couple is considered to be playing an important role for carbohydrate oxidation because it has been reported to be strongly dependent upon the hydroxide concentration. Hydroxyl radicals are probably formed in the $\mathrm{OH}$ oxidation at $\mathrm{Cu}$ (III) catalytic centre during the potential window, which react with the organic molecules through the abstraction of a hydrogen atom from the carbon in a position with respect to -OH group [32]. Large surface area and strong electron transfer rate from electrode to glucose are two commonly important issues responsible for the excellent sensing properties of nonenzymatic glucose sensors. As for as-obtained $\mathrm{CuO} / \mathrm{Ti}$ electrode, more accessible reaction sites provided by the high-density nanoscale "maize cob-like" $\mathrm{CuO}$ building units may generate more $\mathrm{Cu}(\mathrm{III})$ species for the adsorption and reaction of glucose molecules, while abundant spacing among the maize-like building units may facilitate the fast diffusion of glucose molecules. In addition, more electron transfer passages and lower charge transfer 


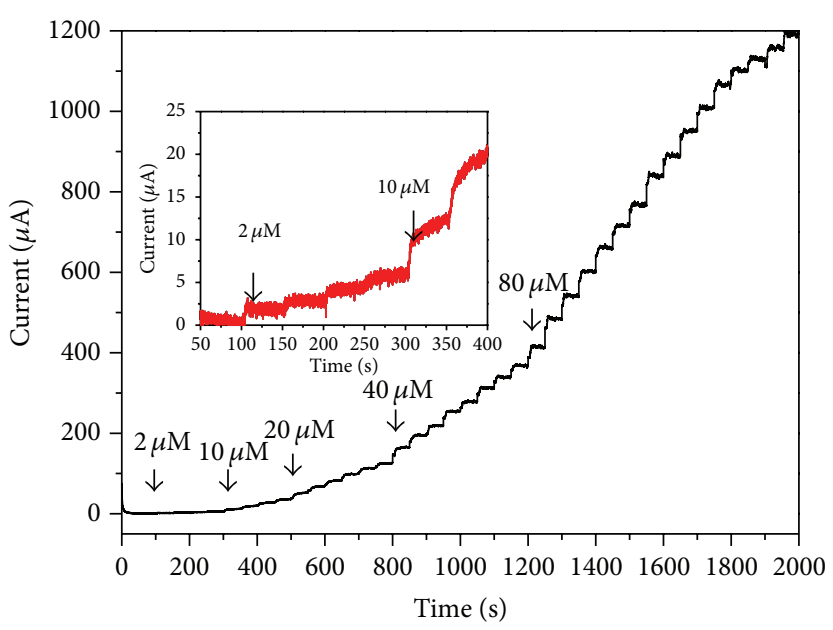

(a)

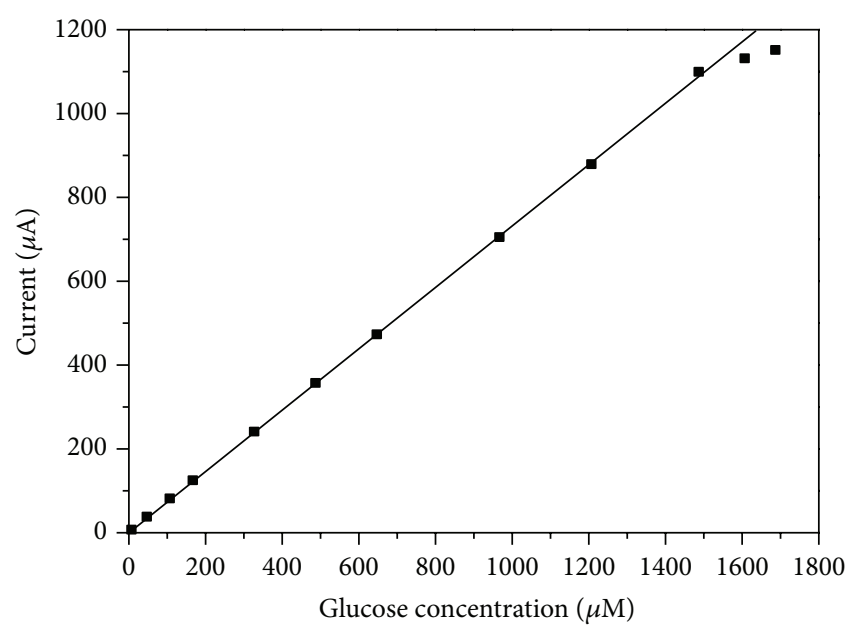

(b)

Figure 3: (a) Amperometric responses of the $\mathrm{CuO}$ film electrode to successive addition of glucose at applied potential of $+0.58 \mathrm{~V}$ in $0.1 \mathrm{M}$ $\mathrm{NaOH}$; (b) the calibration curve for the amperometric responses of $\mathrm{CuO}$ film electrode. Inset of (a) displays the response to $2 \mu \mathrm{M}$ and $10 \mu \mathrm{m}$ glucose of the $\mathrm{CuO}$ film electrode.

resistance for hierarchical $\mathrm{CuO}$ micro/nanostructures can promote the shuttle of electron between glucose and the working electrode. In a way, in situ fabrication of maize coblike $\mathrm{CuO}$ on Ti substrate may further ensure the direct and fast electron transfer between electrode and electrolyte.

The long-term stability of glucose sensor is a critical factor in practical detection application. The reproducibility and stability of the as-obtained sensor were evaluated by the comparison of the sensitivity of different electrodes. Six different electrodes were made under the same fabrication condition and their current responses to glucose at $+0.58 \mathrm{~V}$ were investigated. The relative standard deviation (RSD) is $3.5 \%$, which shows highly reproducible. Thirty successive measurements of glucose on one electrode yield an RSD of $4.1 \%$, demonstrating that the sensor is stable. It indicates that the glucose sensor based on $\mathrm{CuO} / \mathrm{Ti}$ electrode has good reproductivity and long-term stability. The excellent longterm storage stability of the electrode can be attributed to the strong adhesion of $\mathrm{CuO} / \mathrm{Ti}$ substrate, as well as the chemical stability of $\mathrm{CuO}$ in basic solution.

\section{Conclusion}

We have successfully synthesized $\mathrm{CuO}$ films on the $\mathrm{Ti}$ substrate by a simple, rapid, and reproducible method. For the first time, we used $\mathrm{CuO} / \mathrm{Ti}$ as working electrode to detect glucose which is inexpensive and convenient. The novel amperometric glucose sensor based on the $\mathrm{CuO} / \mathrm{Ti}$ electrode shows high sensitivity, low detection limit, good stability, reproducibility, and fast response time. The $\mathrm{CuO} / \mathrm{Ti}$ electrode is easily fabricated and afforded an excellent platform for glucose sensor.

\section{Conflict of Interests}

The authors declare that there is no conflict of interests regarding the publication of this paper.

\section{Acknowledgments}

This work was supported by the Natural Science Foundation of Henan Province (nos. 122300410214; 132102210048). The authors would like to thank Professor X. T. Huang for his guidance and help at Central China Normal University.

\section{References}

[1] A. Safavi, N. Maleki, and E. Farjami, "Fabrication of a glucose sensor based on a novel nanocomposite electrode," Biosensors and Bioelectronics, vol. 24, no. 6, pp. 1655-1660, 2009.

[2] E. Ohashi and I. Karube, "Development of a thin membrane glucose sensor using $\beta$-type crystalline chitin for implantable biosensor," Journal of Biotechnology, vol. 40, no. 1, pp. 13-19, 1995.

[3] D. R. Shankaran, K. V. Gobi, and N. Miura, "Recent advancements in surface plasmon resonance immunosensors for detection of small molecules of biomedical, food and environmental interest," Sensors and Actuators, B: Chemical, vol. 121, no. 1, pp. 158-177, 2007.

[4] B. J. White and H. J. Harmon, "Novel optical solid-state glucose sensor using immobilized glucose oxidase," Biochemical and Biophysical Research Communications, vol. 296, no. 5, pp. 10691071, 2002.

[5] S. Cherevko and C. Chung, "Gold nanowire array electrode for non-enzymatic voltammetric and amperometric glucose detection," Sensors and Actuators, B: Chemical, vol. 142, no. 1, pp. 216223, 2009. 
[6] K. M. El Khatib and R. M. Abdel Hameed, "Development of $\mathrm{Cu}_{2} \mathrm{O} /$ Carbon Vulcan XC-72 as non-enzymatic sensor for glucose determination," Biosensors and Bioelectronics, vol. 26, no. 8, pp. 3542-3548, 2011.

[7] T. Watanabe and Y. Einaga, "Design and fabrication of nickel microdisk-arrayed diamond electrodes for a non-enzymatic glucose sensor based on control of diffusion profiles," Biosensors and Bioelectronics, vol. 24, no. 8, pp. 2684-2689, 2009.

[8] C. Xia and W. Ning, "A novel non-enzymatic electrochemical glucose sensor modified with FeOOH nanowire," Electrochemistry Communications, vol. 12, no. 11, pp. 1581-1584, 2010.

[9] M. Q. Guo, H. S. Hong, X. N. Tang, H. D. Fang, and X. $\mathrm{H}$. Xu, "Ultrasonic electrodeposition of platinum nanoflowers and their application in nonenzymatic glucose sensors," Electrochimica Acta, vol. 63, pp. 1-8, 2012.

[10] L.-M. Lu, L. Zhang, F.-L. Qu et al., "A nano-Ni based ultrasensitive nonenzymatic electrochemical sensor for glucose: enhancing sensitivity through a nanowire array strategy," Biosensors and Bioelectronics, vol. 25, no. 1, pp. 218-223, 2009.

[11] J. Wang, D. F. Thomas, and A. Chen, "Nonenzymatic electrochemical glucose sensor based on nanoporous PtPb networks," Analytical Chemistry, vol. 80, no. 4, pp. 997-1004, 2008.

[12] H. B. Noh, K. S. Lee, P. Chandra, M. S. Won, and Y. B. Shim, "Application of a Cu-Co alloy dendrite on glucose and hydrogen peroxide sensors," Electrochimica Acta, vol. 61, pp. 36-43, 2012.

[13] Y. Zhao, J. Z. Zhao, D. C. Ma et al., "Synthesis, growth mechanism of different $\mathrm{Cu}$ nanostructures and their application for non-enzymatic glucose sensing," Colloids and Surfaces A: Physicochemical and Engineering Aspects, vol. 409, pp. 105-111, 2012.

[14] Y. Mu, D. Jia, Y. He, Y. Miao, and H. L. Wu, "Nano nickel oxide modified non-enzymatic glucose sensors with enhanced sensitivity through an electrochemical process strategy at high potential," Biosensors and Bioelectronics, vol. 26, no. 6, pp. 29482952, 2011.

[15] X. Kang, Z. Mai, X. Zou, P. Cai, and J. Mo, "A sensitive nonenzymatic glucose sensor in alkaline media with a copper nanocluster/multiwall carbon nanotube-modified glassy carbon electrode," Analytical Biochemistry, vol. 363, no. 1, pp. 143-150, 2007.

[16] J. S. Ye, Y. Wen, W. D. Zhang, L. M. Gan, G. Q. Xu, and F. Sheu, "Nonenzymatic glucose detection using multi-walled carbon nanotube electrodes," Electrochemistry Communications, vol. 6 , no. 1, pp. 66-70, 2004.

[17] J. B. Reitz and E. I. Solomon, "Propylene oxidation on copper oxide surfaces: Electronic and geometric contributions to reactivity and selectivity," Journal of the American Chemical Society, vol. 120, no. 44, pp. 11467-11478, 1998.

[18] A. Chowdhuri, V. Gupta, K. Sreenivas, R. Kumar, S. Mozumdar, and P. K. Patanjali, "Response speed of $\mathrm{SnO}_{2}$-based $\mathrm{H}_{2} \mathrm{~S}$ gas sensors with $\mathrm{CuO}$ nanoparticles," Applied Physics Letters, vol. 84, no. 7, pp. 1180-1182, 2004.

[19] C.-T. Hsieh, J.-M. Chen, H.-H. Lin, and H.-C. Shih, "Field emission from various $\mathrm{CuO}$ nanostructures," Applied Physics Letters, vol. 83, no. 16, pp. 3383-3385, 2003.

[20] X. P. Gao, J. L. Bao, G. L. Pan et al., "Preparation and electrochemical performance of polycrystalline and single crystalline $\mathrm{CuO}$ nanorods as anode materials for $\mathrm{Li}$ ion battery," Journal of Physical Chemistry B, vol. 108, no. 18, pp. 5547-5551, 2004.

[21] M. U. Anu Prathap, B. Kaur, and R. Srivastava, "Hydrothermal synthesis of $\mathrm{CuO}$ micro-/nanostructures and their applications in the oxidative degradation of methylene blue and nonenzymatic sensing of glucose $/ \mathrm{H}_{2} \mathrm{O}_{2}$," Journal of Colloid and Interface Science, vol. 370, no. 1, pp. 144-154, 2012.

[22] P. Zhang, L. Zhang, G. C. Zhao, and F. Feng, "A highly sensitive nonenzymatic glucose sensor based on $\mathrm{CuO}$ nanowires," Microchimica Acta, vol. 176, no. 3-4, pp. 411-417, 2012.

[23] E. Reitz, W. Z. Jia, M. Gentile, Y. Wang, and Y. Lei, "CuO nanospheres based nonenzymatic glucose sensor," Electroanalysis, vol. 20, no. 22, pp. 2482-2486, 2008.

[24] Z. H. Ibupoto, K. Khun, V. Beni, X. J. Liu, and M. Willander, "Synthesis of novel $\mathrm{CuO}$ nanosheets and their non-enzymatic glucose sensing applications," Sensors, vol. 13, no. 6, pp. 79267938, 2013.

[25] X. Wang, C. G. Hu, H. Liu, G. J. Du, X. S. He, and Y. Xi, "Synthesis of $\mathrm{CuO}$ nanostructures and their application for nonenzymatic glucose sensing," Sensors and Actuators, B: Chemical, vol. 144, no. 1, pp. 220-225, 2010.

[26] L.-L. Tian and B.-T. Liu, "Fabrication of $\mathrm{CuO}$ nanosheets modified $\mathrm{Cu}$ electrode and its excellent electrocatalytic performance towards glucose," Applied Surface Science, vol. 283, pp. 947-953, 2013.

[27] T. K. Huang, K. W. Lin, S. P. Tung et al., "Glucose sensing by electrochemically grown copper nanobelt electrode," Journal of Electroanalytical Chemistry, vol. 636, no. 1-2, pp. 123-127, 2009.

[28] X. Zhang, A. X. Gu, G. F. Wang et al., "Fabrication of $\mathrm{CuO}$ nanowalls on $\mathrm{Cu}$ substrate for a high performance enzyme-free glucose sensor," CrystEngComm, vol. 12, no. 4, pp. 1120-1126, 2010.

[29] X. Li, J. Liu, X. Ji et al., "Ni/Al layered double hydroxide nanosheet film grown directly on $\mathrm{Ti}$ substrate and its application for a nonenzymatic glucose sensor," Sensors and Actuators B: Chemical, vol. 147, no. 1, pp. 241-247, 2010.

[30] G. Y. Liu, B. Z. Zheng, Y. S. Jiang et al., "Improvement of sensitive $\mathrm{CuO}$ NFs-ITO nonenzymatic glucose sensor based on in situ electrospun fiber," Talanta, vol. 101, pp. 24-31, 2012.

[31] D. Ye, G. Liang, H. Li et al., "A novel nonenzymatic sensor based on $\mathrm{CuO}$ nanoneedle/graphene/carbon nanofiber modified electrode for probing glucose in saliva," Talanta, vol. 116, pp. 223230, 2013.

[32] I. G. Casella, T. R. I. Cataldi, A. Guerrieri, and E. Desimoni, "Copper dispersed into polyaniline films as an amperometric sensor in alkaline solutions of amino acids and polyhydric compounds," Analytica Chimica Acta, vol. 335, no. 3, pp. 217-225, 1996. 

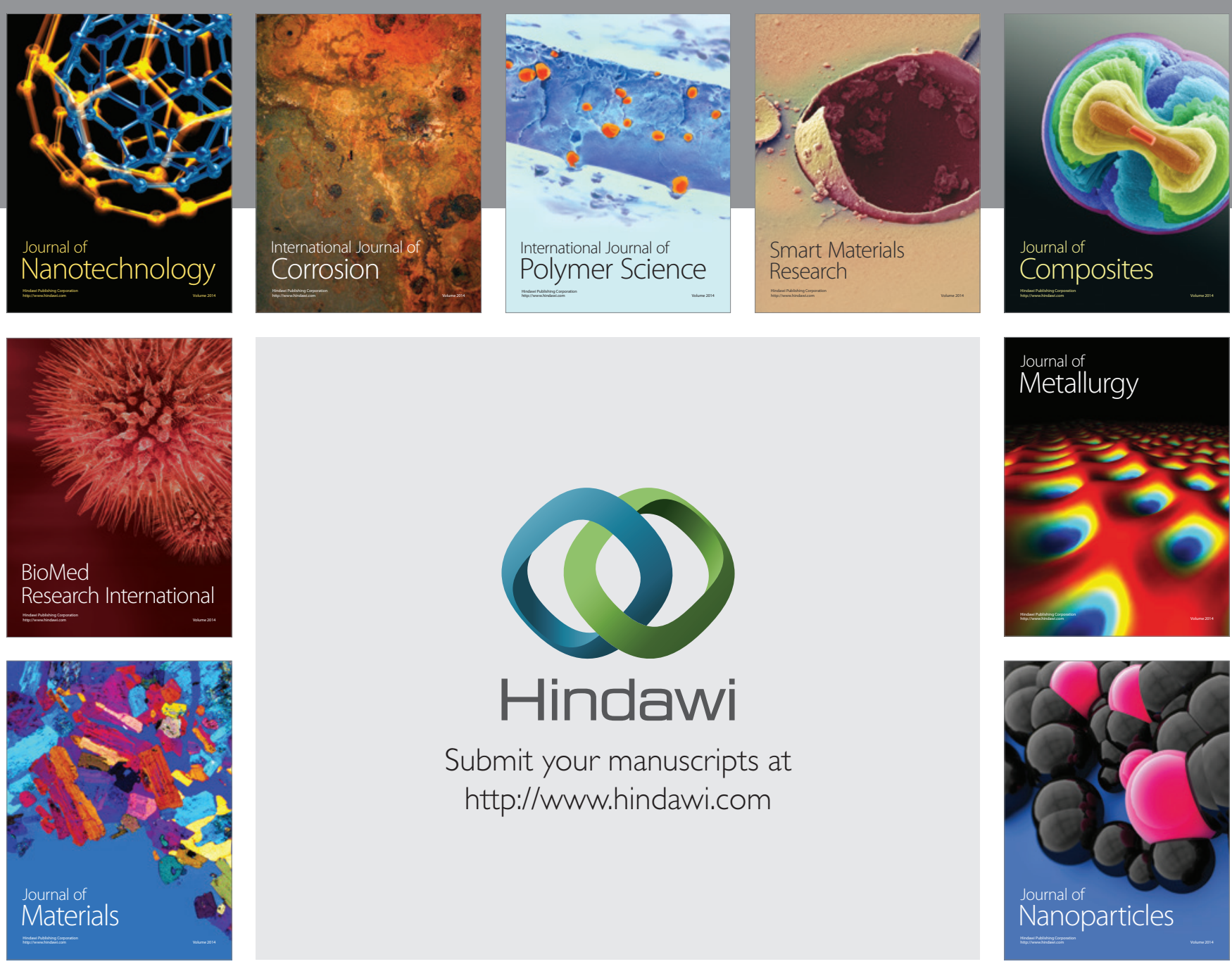

Submit your manuscripts at http://www.hindawi.com
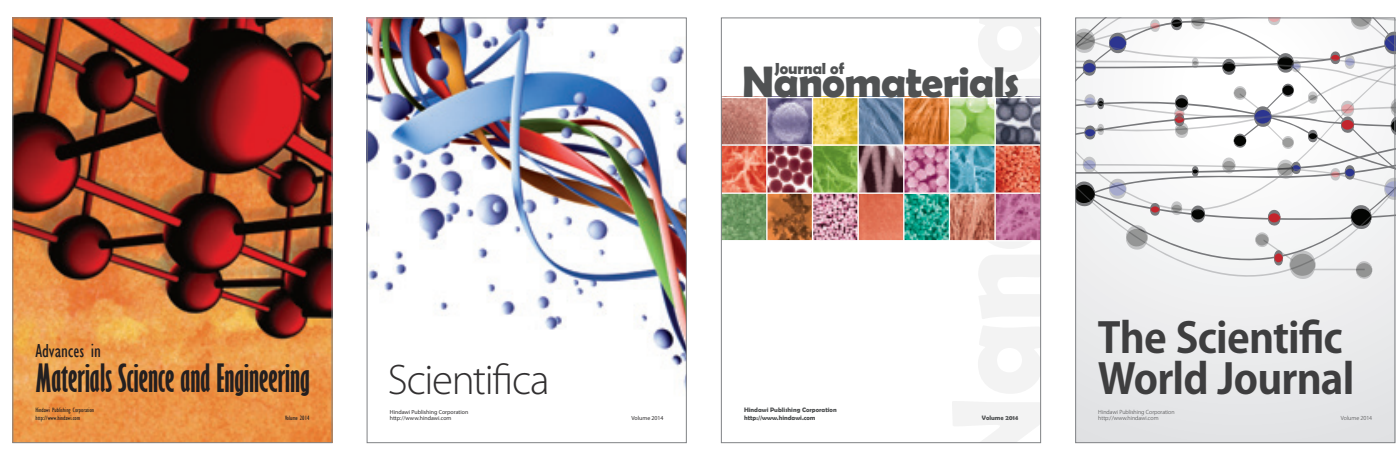

\section{The Scientific World Journal}
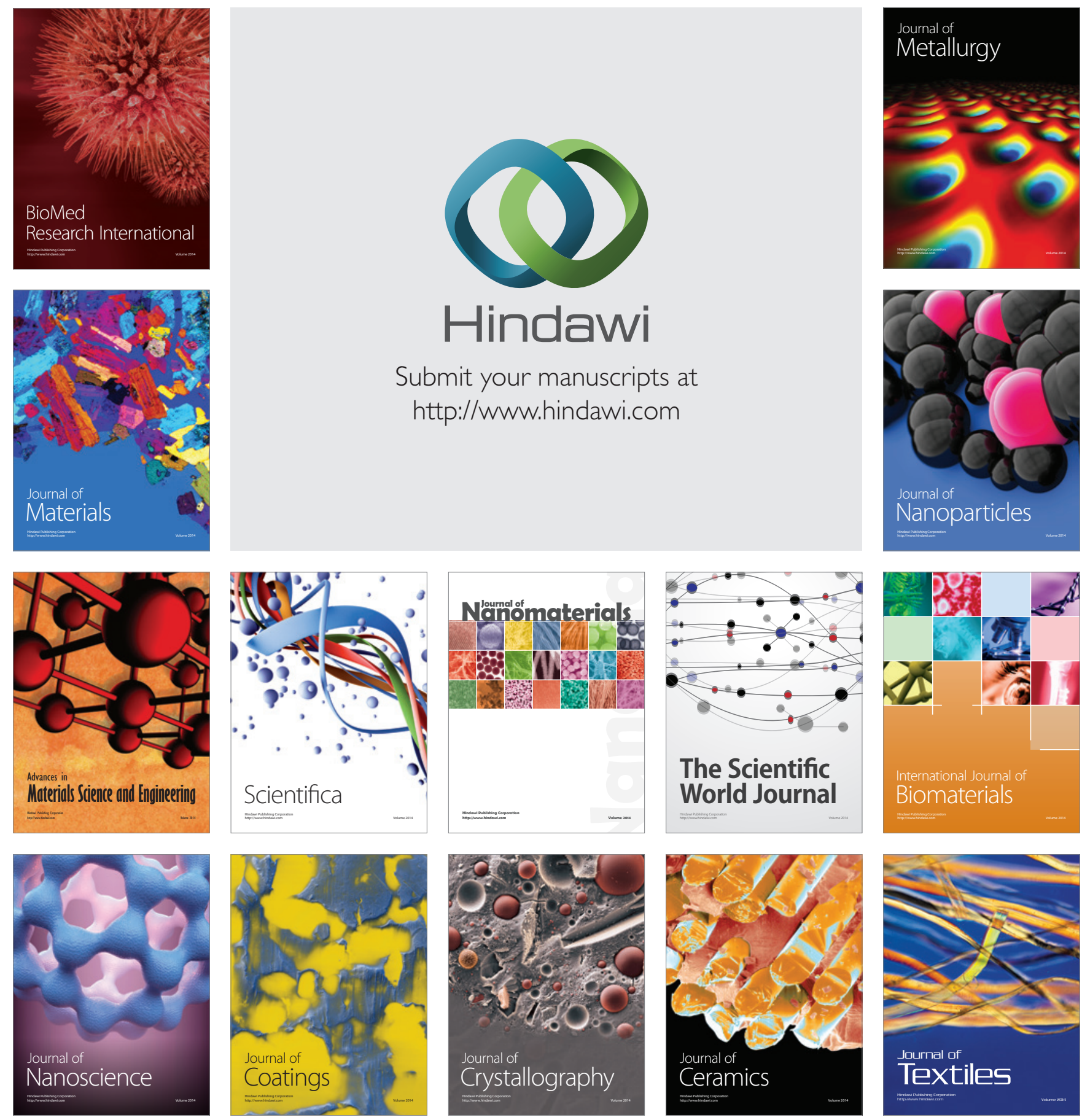